\title{
Online Detection of Partial Discharges in Medium and High Voltage Cable Accessories by Acoustic Emissions
}

\author{
Pau Casals Torrens ${ }^{1}$, Adrián González Parada ${ }^{2}$, Ricard Bosch Tous ${ }^{3}$
}

\author{
${ }^{1,3}$ Universitat Politècnica de Catalunya Departamento de Ingeniería Eléctrica, C. Jordi Girona, 1-3. 08034 Barcelona - España \\ ${ }^{2}$ DICIS Universidad de Guanajuato México, Lascuráin de Retana No. 5, Col. Centro C.P. 36000 Guanajuato, Gto., México
}

Email: p.casals@upc.edu,gonzaleza@salamanca.ugto.mx, bosch@ee.upc.edu

Received: November $24^{\text {th }}, 2017$

Accepted:. November $30^{\text {th }}$, 2017

Published:. December $30^{\text {th }}, 2017$

Copyright (02016 by authors and Institute of Technology Galileo of Amazon (ITEGAM). This work is licensed under the Creative Commons Attribution International License (CC BY 4.0).

http://creativecommons.org/licenses/by/4.0/ (c) (1) (2)(2) Open Acteres

\begin{abstract}
The acoustic waves caused by partial discharges inside a dielectric can be detected by sensors of acoustic emissions (EA) and analyzed in the time domain. The experimental results that are presented, demonstrate the capability of online detection of these sensors, in the vicinity of a cable, splice or terminal accessory. The EA sensors are immune to electromagnetic interference, are a non-destructive detection method and guarantee galvanic decoupling from the voltage network; This technique of detection of partial discharges can be applied as a test method for preventive or predictive maintenance (maintenance based on the condition), in equipment or installations of medium and high voltage in service and represents an alternative to electrical detection systems, conventional or not, which continue to be based on the detection of current pulses.
\end{abstract}

Keywords: Acoustic waves, discharges, medium and high voltage.

\section{Detección en Línea de Descargas Parciales en Accesorios de Cables de Media y Alta Tensión mediante Emisiones Acústicas}

\section{RESUMEN}

Las ondas acústicas provocadas por las descargas parciales en el interior de un dieléctrico pueden ser detectadas por sensores de emisiones acústicas (EA) y analizadas en el dominio del tiempo. Los resultados experimentales que se presentan, evidencia la capacidad de detección en línea de estos sensores, en el entorno próximo a un accesorio de cable, empalme o terminal. Los sensores EA son inmunes a las interferencia electromagnéticas, son un método de detección no destructivo y garantizan desacople galvánico respecto a la red de tensión; esta técnica de detección de descargas parciales puede ser aplicada como método de prueba para mantenimiento preventivo o predictivo (mantenimiento basado en la condición), en equipos o instalaciones de media y alta tensión en servicio y representa una alternativa a los sistemas eléctricos de detección, convencionales o no, que continúan basándose en la detección del impulsos de corriente.

Palabras Claves: Ondas acústicas, descargas, media y alta tensión.

\section{INTRODUCCIÓN}

La fiabilidad y continuidad del suministro eléctrico, en las redes subterráneas de distribución y transporte, en especial en alta tensión, se ve afectada por la elevada tasa de fallo de accesorios (empalmes o terminales), en comparación a los fallos de los propios cables [1-3]. Esto esencialmente se debe a que finalizada la fabricación de los cables y antes de su instalación, están sometidos a rigurosos ensayos de calidad, tanto de rigidez dieléctrica, como de descargas parciales, que aseguran la ausencia inicial de defectos en los mismos. Los accesorios en cambio, no pueden ser probados como conjunto al $100 \%$ [4,5]; después de la fabricación y ensayo de sus partes, los accesorios sufren un proceso de manipulación durante el montaje, que también afecta la terminación del cable. Todo este proceso está propenso a errores y daños que difícilmente pueden ser detectados en los ensayos posteriores a la instalación [4], debido a las limitaciones de los ensayos de campo: menor rigurosidad que los de laboratorio (nivel de tensión y sensibilidad inferiores) y ensayo de 
descargas parciales no requerido [4], que en consecuencia pocas veces se realiza.

En este sentido toda nueva tecnología que pueda contribuir a garantizar dicha fiabilidad o a facilitar información, que permita con antelación, identificar potenciales situaciones de fallo en los accesorios, sin duda reviste un especial interés ya que permitiría efectuar de manera planificada, los descargos y las operaciones preventivas de mantenimiento en las instalaciones. Sin la urgencia, el coste económico y desprestigio del servicio, que provocan las interrupciones debidas a fallos "imprevistos".

Los métodos eléctricos ofrece alta fiabilidad cuando se usa en laboratorios apantallados o zonas libres de interferencias, pero en pruebas de campo resultan costosos y muy sensibles a las interferencias de radio frecuencia (RF); lo cual abre un espacio de oportunidades a sistemas no convencionales, no eléctricos, como el sistema de nuestro estudio, basado exclusivamente en EA, cuyos resultados y prestaciones se presentan.

\section{DETECCIÓN DE DESCARGAS PARCIALES (DP)}

El análisis de los diferentes métodos existentes para cables o accesorios, lo limitaremos y resumiremos a los siguientes dos grandes grupos, de acuerdo con el principio sensor aplicado: capacitivo)

- sensores eléctricos (inductivo, capacitivo, inductivo-

- sensores acústicos.

\section{II.1. DETECCIÓN CON SENSORES ELÉCTRICOS}

Sea sistema convencional (normalizado IEC) [6], o no, se basan en la detección de impulsos de corriente y de tensión. [7].

Desventajas:

- En pruebas de campo son muy sensibles a las interferencias de RF, al no estar apantallados.

- Las interferencias son reducidas o eliminadas por sofisticados sistemas de discriminación (hardware o software), que pueden filtrar o enmascarar las descargas.

- Los inductivos están limitados por el tipo constructivo de la pantalla del cable.

- La sensibilidad de los capacitivos está limitada por su bajo valor de capacidad de acoplamiento respecto a la capacidad del objeto bajo ensayo (accesorio + cable).

- El tratamiento de la respuesta del sensor, en la mayoría de casos se realiza con equipos de medida de descargas parciales.

- Son costosos, lo cual dificulta su uso generalizado y su instalación en cada accesorio.

Ventajas:

- Permiten conocer el nivel y tipo de descarga.

- Se pueden complementar con sistemas de localización de descargas.

- El uso de doble sensor permite auto calibración y analizar la dirección de descargas en empalmes.

- Existen sistemas comerciales para alta tensión (AT).

\section{II.2. DETECCIÓN CON SENSORES ACÚSTICOS}

Es una filosofía diferente, basada en la detección de variaciones de presión en la superficie del aislamiento, que ofrece solución al problema de detección de descargas parciales en accesorios, en línea y en campo, sin presentar las mismas desventajas de los sensores eléctricos [7].

Desventajas:

- Actualmente la mayoría de sensores y sistemas de detección están en desarrollo.

- No se comercializan equipos para aplicaciones específicas en accesorios de cables de AT.

- Reducción de sensibilidad debido a la atenuación con la distancia y con las elevadas temperaturas que puede alcanzar el material de aislamiento.

- Los detectores de uso externo en cables de media tensión (MT), tienen una sensibilidad limitada por las interfases de aire (baja transmisión acústica) que hay en el interior de los cables o empalmes.

- No permiten conocer el nivel de descarga.

- La calibración es compleja.

Ventajas:

- Insensibles a las interferencias de radio frecuencia (RF), no requieren pantalla.

- Insensibles a los campos electromagnéticos.

- Es un método de ensayo no destructivo

- Sensores de alta sensibilidad, sólo requieren amplificación y visualización con osciloscopio digital, sin tratamientos complejos de señal.

- Tienen amplio espectro de frecuencia.

- El tipo constructivo de pantalla del cable no impide su aplicación.

- Alta robustez mecánica.

- Alta resistividad eléctrica que ofrece separación galvánica respecto al equipo ensayado.

- Pueden ofrecer precios de comercialización menores, que permitan su uso generalizado y su instalación unitaria por empalme de AT.

\section{CONCEPTOS DE LA DETECCIÓN ACÚSTICA DE DP}

Debido a las pocas publicaciones sobre el fenómeno acústico, que provoca las descargas parciales en cables, hemos considerado conveniente incluir algunos conceptos que permitan comprender dicho fenómeno y su detección en el interior de un cable o su respectivo accesorio. La DP representa una pequeña explosión, con colisiones entre moléculas, la cual excita la emisión de ondas de presión (acústicas) [8, 9 y 10] que se propagan a través del material de aislamiento. Estas ondas mecánicas pueden ser detectadas por sensores de emisiones acústicas (EA), sensibles a los cambios de presión que se producen en la superficie del aislamiento, que envuelve la cavidad o defecto, especialmente en las finas capas semiconductoras.

Debido a las pocas publicaciones sobre el fenómeno acústico, que provoca las descargas parciales en cables, hemos considerado conveniente incluir algunos conceptos que permitan comprender dicho fenómeno y su detección en el interior de un cable o su respectivo accesorio. La DP representa una pequeña explosión, con colisiones entre moléculas, la cual excita la emisión de ondas de presión (acústicas) [8, 9 y 10] que se propagan a través del material de aislamiento. Estas ondas mecánicas pueden ser detectadas por sensores de emisiones acústicas (EA), sensibles a los cambios de presión que se producen en la superficie del aislamiento, que envuelve la cavidad o defecto, especialmente en las finas capas semiconductoras. 
La energía que transfiere una descarga, en un defecto tipo cavidad, se puede calcular por la diferencia entre la energía almacenada antes y después de la descarga parcial. La magnitud de la energía liberada (W), en función del valor eficaz de la tensión de excitación de la descarga (Vi) y de la carga transferida (qc), se puede expresar como:

$$
\mathrm{W} \cong(1 / \sqrt{2}) \cdot \mathrm{q}_{\mathrm{c}} \cdot \mathrm{V}_{\mathrm{i}(\mathrm{mss})}=0,707 \cdot \mathrm{q}_{\mathrm{c}} \cdot \mathrm{V}_{\mathrm{i}(\mathrm{ms})}
$$

Esta energía puede presentar órdenes de magnitud de $10^{-}$ 9 a $10^{-5} \mathrm{~J}[11,12]$, varía con el espesor de aislamiento y provoca localmente diferentes efectos. Entre ellos la radiación de energía en forma de ondas acústicas.

Si simulamos y analizamos, mediante elementos finitos, el campo eléctrico y su comportamiento en una cavidad de $1 \mathrm{~mm}$ de diámetro, en el interior de un aislamiento de $18 \mathrm{~mm}$ de espesor, de un cable de alta tensión tipo XLPE 1x400 mm2 Al, $76 / 132 \mathrm{kV}$. La simulación nos permitirá conocer que la energía que almacena dicha cavidad, antes de descargar, cuando el cable trabaja a su tensión de $76 \mathrm{kV}$, varía de $25,6 \times 10-5 \mathrm{~J}$ a 5,59x10-5 J, entre la zona de máximo gradiente y mínimo gradiente, respectivamente [7].

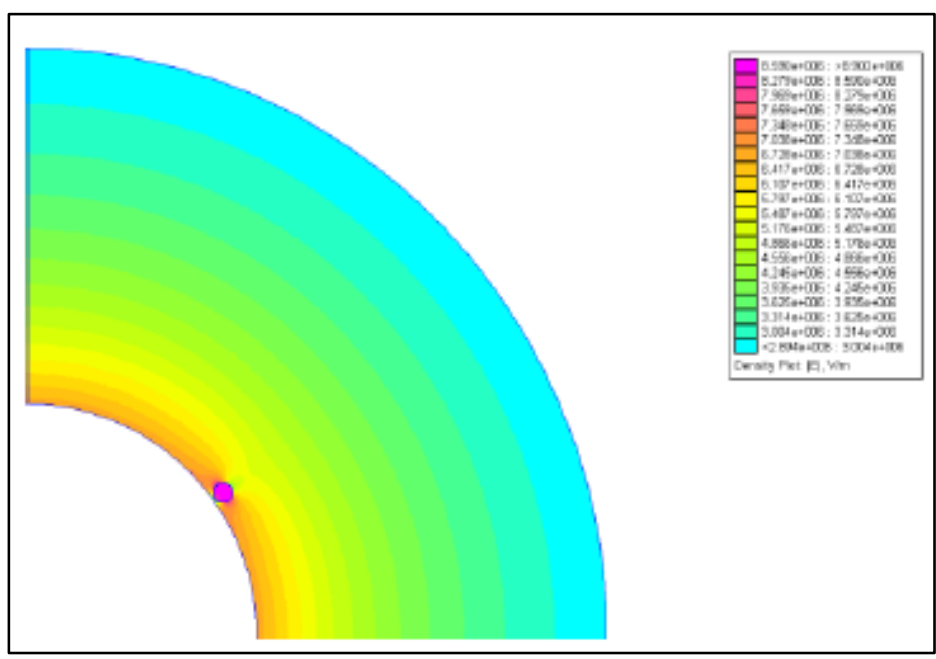

Figura 1: Cavidad adyacente a la capa semiconductora interior (máximo gradiente).

Fuente: Autores, (2017).

Utilizando conceptos descritos en libros de acústica [13, 14], el sonido de la descarga se propaga a través del medio, a velocidad (c), de forma mecánica, mediante el movimiento de ondas longitudinales, que provocan cambios locales de presión $(p)$, densidad ( $\rho$ ) y desplazamiento de las moléculas del medio. La ecuación de propagación de onda, para campos de presión esféricamente simétricos, se representa por la ecuación:

$$
\nabla^{2} \mathrm{p}=\frac{1}{\mathrm{c}^{2}} \frac{\partial^{2} \mathrm{p}}{\partial \mathrm{t}^{2}}
$$

Los pulsos de descargas parciales tienen duraciones muy cortas, que oscilan desde $1 \mathrm{~ns}$ a cientos de ns [8] [11] [15], esto provoca que el espectro de frecuencia de las ondas acústicas generadas sea muy amplio, en el rango de los ultrasonidos, desde varios $\mathrm{kHz}$ hasta los $\mathrm{MHz}$.

Si consideramos la descarga parcial en una cavidad, como una fuente puntual, simple, generalmente muy pequeña, inferior a $1 \mu \mathrm{m} 3$; cuando inicia su actividad, emite ondas acústicas esféricas, de simetría radial, como se representa en la figura 2.

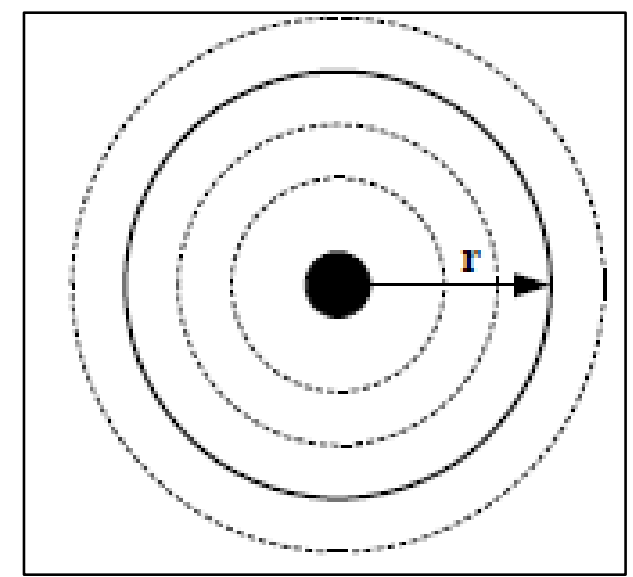

Figura 2: Ondas acústicas de fuente puntual.

Fuente: Autores, (2017).

En el interior de un empalme de cable, la descarga parcial se produce siguiendo la dirección radial de máxima variación del campo eléctrico. Esta misma dirección es la más corta en la propagación de las ondas acústicas esféricas, hasta la frontera con un médio diferente al aislamiento, de acuerdo con la representación de la figura 3 , donde (r) representa el radio que alcanzan.

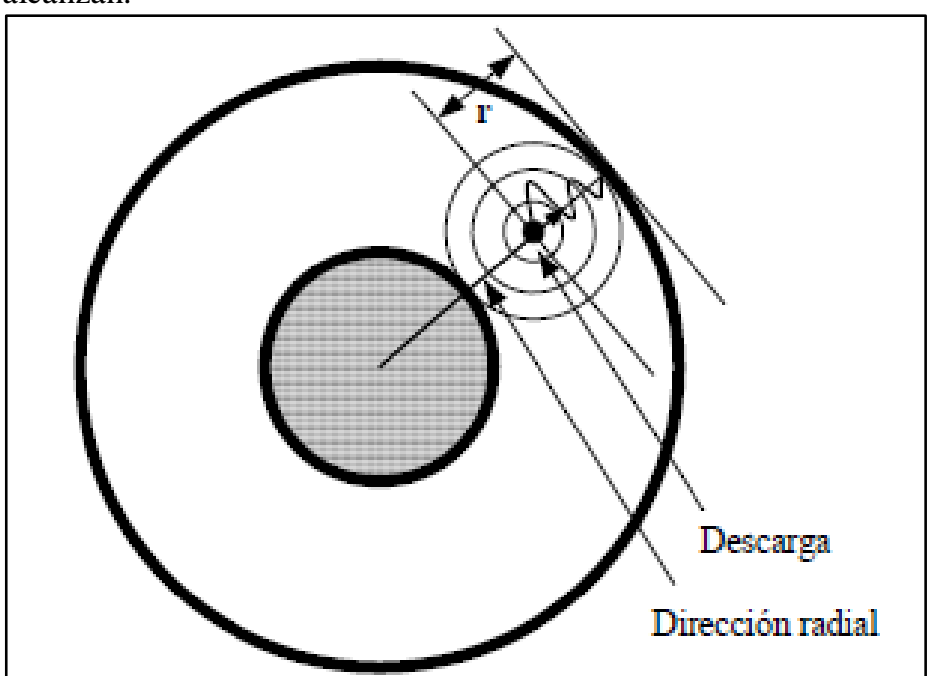

Figura 3: Representación de la propagación radial de una onda acústica.

Fuente: Autores, (2017).

En consecuencia, los frentes de presión de las ondas acústicas lo primero que alcanzan son ambas capas semiconductoras y el conductor, con una diferencia de tiempo que dependerá de la posición de la descarga en el interior del aislamiento. Entre estos medios se produce la refracción o reflexión de dicha onda, en función de la impedancia acústica específica $(Z)$ de los mismos, la cual se define como la razón de la presión acústica del medio (p) y la velocidad de partícula asociada $(\mathrm{V})$ :

$$
\overrightarrow{\mathrm{z}}=\frac{\overrightarrow{\mathrm{p}}}{\overrightarrow{\mathrm{V}}}
$$

que también puede expresarse como:

$$
\overrightarrow{\mathrm{z}}=\rho_{o} c \cos \beta e^{\mathrm{j} \beta}
$$

donde ( $\rho$ ) es la densidad característica del material y $(\beta)$ el ángulo entre la dirección de la presión acústica y la velocidad de partícula en el material. $\beta=0$ cuando la onda es plana.

Los frentes de ondas que siguen direcciones no radiales, como la dirección axial del cable, figura 4 , recorren un camino 
más largo, pero finalmente también alcanzan e inciden oblicuamente sobre las capas semiconductoras y el conductor.

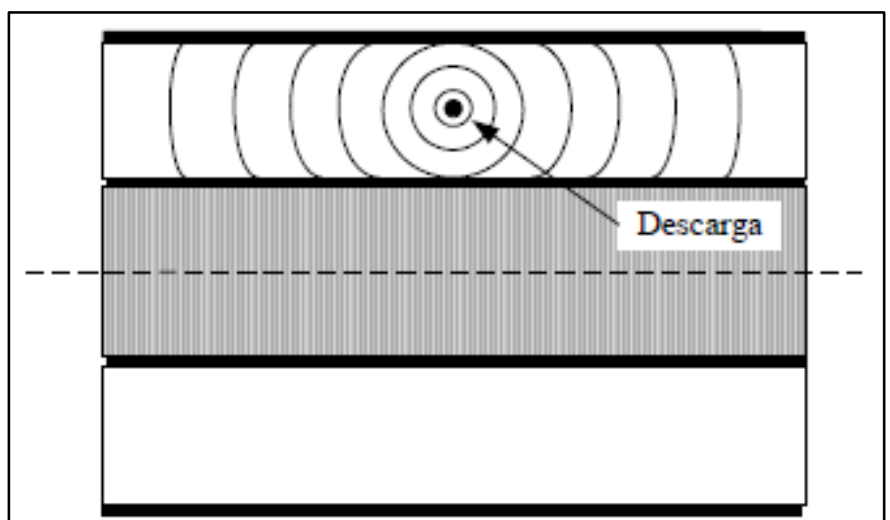

Figura 4: Representación de la propagación axial de ondas acústicas

Fuente: Autores, (2017).

La velocidad longitudinal del sonido en el polietileno, incrementa linealmente con la densidad y puede variar aproximadamente entre 2003 y 1977 m/s [16], dependiendo del grado de curado del material.

En la mayoría de polímeros, comúnmente usados em aislamientos de media y alta tensión, el coeficiente de atenuación se reduce aproximadamente de forma lineal con el incremento del módulo de Young del material [8]. Éste módulo (ecuación 5) nos da una indicación del grado de rigidez del material y su magnitud disminuye con el incremento de temperatura [17].

$$
\mathrm{Y}=\rho \cdot \mathrm{c}^{2}
$$

La fuerte incidencia de la atenuación, es el condicionante que limita a distancias cortas esta técnica de detección; pero a su vez la hace adecuada para el control focalizado en equipos de poca longitud, como los accesorios de cables.

\section{CONFIGURACIÓN DEL SISTEMA ACÚSTICO DE DETECCIÓN}

El fundamento del sistema de detección consiste em incorporar sensores de emisión acústica (EA), externos y próximos al accesorio del cable, figuras 5, 6 y 7, o internos al propio accesorio en zona de potencial 0.

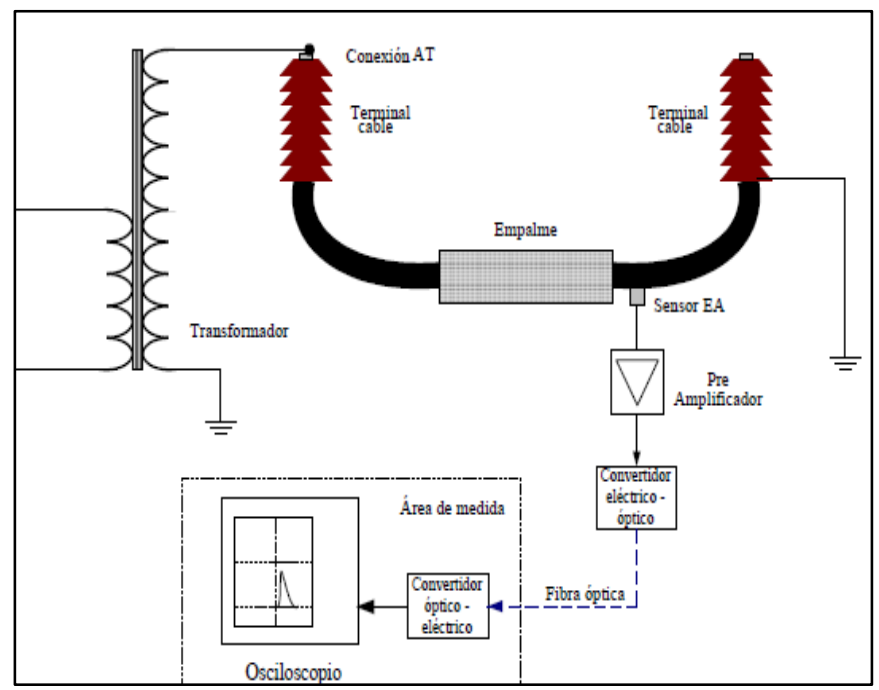

Figura 5: Esquema del sistema de detección acústico, con un sensor colocado próximo a un empalme.

Fuente: Autores, (2017).
El sensor o sensores, recomendable uno en cada extremo, activado por las ondas acústicas, genera una señal de respuesta que se envía a un pre amplificador donde pueden combinarse las diferentes señales.

Los sensores de emisión acústica (EA), ofrecen sensibilidad para asegurar una información permanente (sistema en línea) o periódica (lectura según plan preventivo) de la actividad de PD detectada y en especial de las variaciones de la misma en el tiempo. Pudiendo generar alarmas según los diferentes niveles de actividad, consecuencia de la degradación del accesorio.

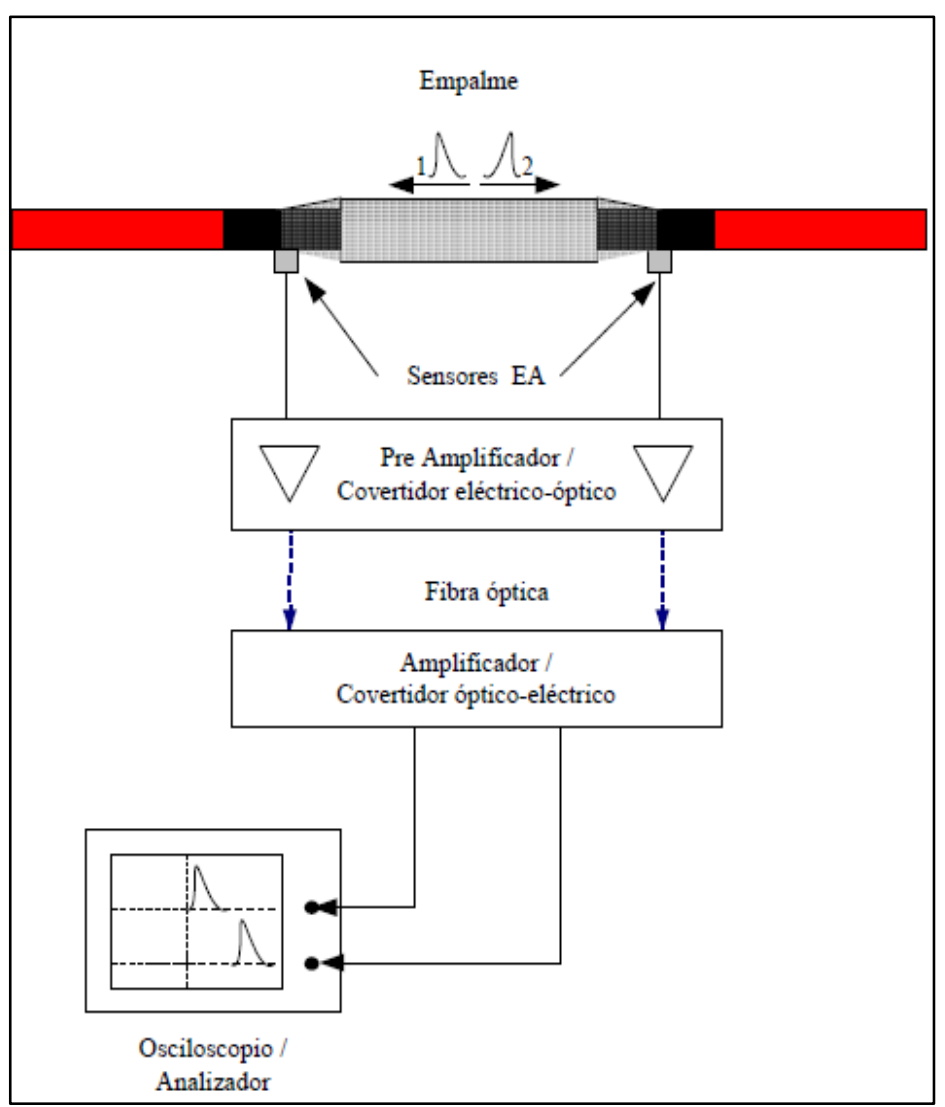

Figura 6: Esquema del sistema de detección acústico, con dos sensores colocados al interior de un empalme.

Fuente: Autores, (2017).

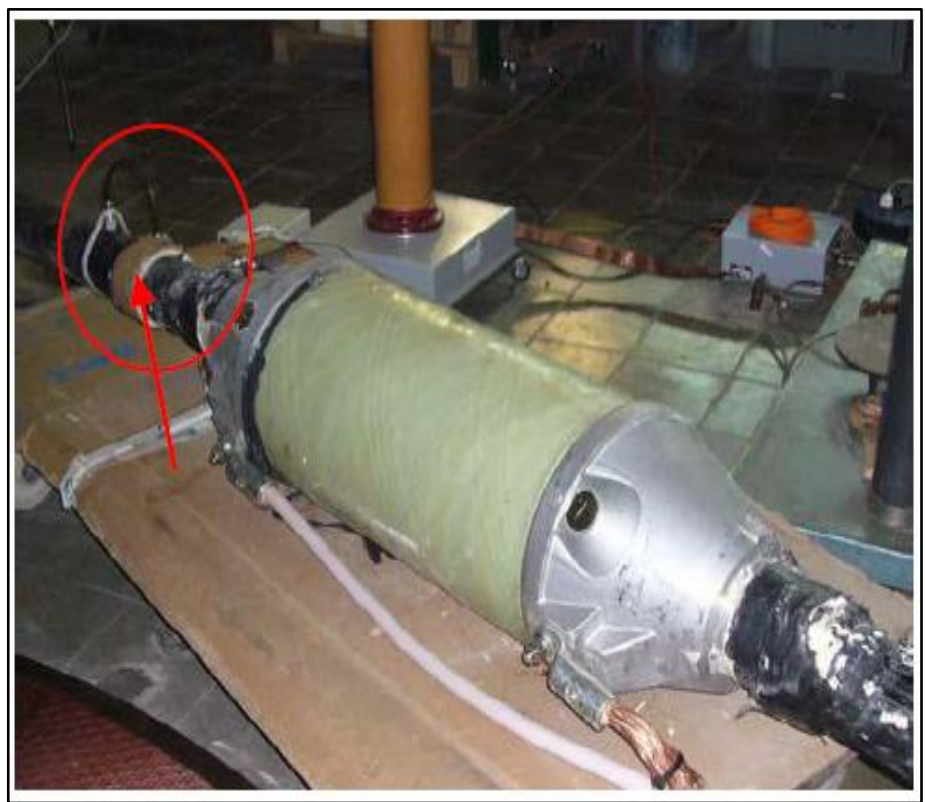

Figura 7: Sensor ubicado próximo a un terminal de AT.

Fuente: Autores, (2017). 
Torrens, Parada \& Tous, ITEGAM-JETIA. Vol. 03, Nº 12, pp 94-99. December, 2017.

Los componentes del sistema son: el sensor EA, un pre amplificador de banda ancha, de 1 y $5000 \mathrm{kHz}$, convertidor electro óptico, salida por fibra óptica y convertidor óptico eléctrico. Los componentes utilizados en nuestro sistema acústico son de diseño propio.

El equipo necesario para medida o análisis, es um osciloscopio digital o un analizador de espectro. Em nuestros ensayos utilizamos un osciloscopio de $200 \mathrm{MHz}$ y $1 \mathrm{GSa} / \mathrm{s}$, pero con un osciloscopio de mayores prestaciones se mejora la capacidad de detección. Esquema en las figuras 5 y 6 .

Dada la importancia que tiene la seguridad, para el técnico que realiza ensayos de alta tensión, está se asegura con la elevada resistividad del sensor y la conexión mediante fibra óptica, garantizando, durante las mediciones, el desacople galvánico entre el sensor y el equipo de medida. La fibra óptica también permite alejar este último del accesorio bajo ensayo.

\section{RESULTADOS EXPERIMENTALES}

Las detecciones se realizaron conjuntamente de forma eléctrica (detector convencional) y acústica, figura 8 . La detección eléctrica nos permitió obtener las magnitudes de descarga en pC y comprobar que el sistema acústico respondía, o no, a la presencia de estas.

Se utilizaron defectos típicos de cable o preparación de accesorios, para simular las descargas parciales. Los mismos se representan en la figura 10, conjuntamente con la respuesta acústica obtenida (trazo amarillo) y la correspondiente transformada rápida de Fourier (FFT, trazo violeta). Los tipos de cables utilizados fueron:

- De alta tensión, de XLPE 76/132kV 1x800mm2 Al.

- De media tensión, de XLPE 12/20 kV 1x240 $\mathrm{mm} 2 \mathrm{Al}$.

En la tabla 1 se resumen los resultados para los tres tipos de defectos.

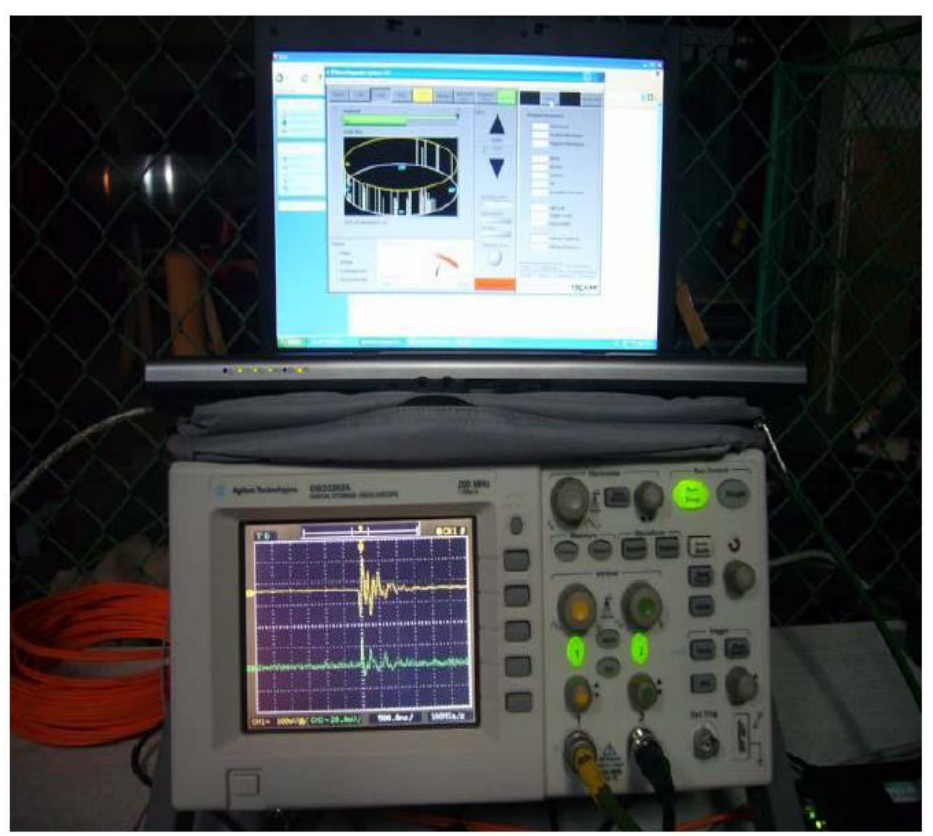

Figura 8: Detección eléctrica y acústica de DP.

Fuente: Autores, (2017).
Tabla 1: Medidas obtenidas.

\begin{tabular}{|c|c|c|c|c|}
\hline $\begin{array}{c}\text { Tipo } \\
\text { Defecto }\end{array}$ & $\begin{array}{c}\text { Tensión } \\
\mathbf{( k V )}\end{array}$ & $\begin{array}{c}\text { Medida } \\
\text { DP } \\
\text { (pC) }\end{array}$ & $\begin{array}{c}\text { Medida } \\
\text { acústica } \\
(\mathbf{m V})\end{array}$ & $\begin{array}{c}\text { Ubicación del } \\
\text { sensior }\end{array}$ \\
\hline $\mathrm{a}$ & 70 & 40 & 136 & $\begin{array}{l}\text { Sobre semicon. a } \\
\text { 25cm del defecto }\end{array}$ \\
\hline $\mathrm{a}$ & 70 & 80 & 164 & $\begin{array}{l}\text { Sobre semicon. a } \\
50 \mathrm{~cm} \text { del defecto }\end{array}$ \\
\hline $\mathrm{b}$ & 12 & 40 & 3,68 & $\begin{array}{l}\text { Sobre semicon. a } \\
50 \mathrm{~cm} \text { del defecto }\end{array}$ \\
\hline $\mathrm{b}$ & 14 & 50 & 5,6 & $\begin{array}{l}\text { Sobre semicon. a } \\
50 \mathrm{~cm} \text { del defecto }\end{array}$ \\
\hline $\mathrm{b}$ & 17 & 70 & 10 & $\begin{array}{l}\text { Sobre cubierta a } \\
50 \mathrm{~cm} \text { del defecto }\end{array}$ \\
\hline $\mathrm{c}$ & 8,4 & 60 & 5,6 & $\begin{array}{l}\text { Sobre semicon. a } \\
10 \mathrm{~cm} \text { del defecto }\end{array}$ \\
\hline $\mathrm{c}$ & 8,2 & 64 & $\begin{array}{c}\text { No } \\
\text { detecta }\end{array}$ & $\begin{array}{l}\text { Sobre semicon. a } \\
\text { 90cm del defecto }\end{array}$ \\
\hline $\mathrm{c}$ & 16,2 & 80 & 2 & $\begin{array}{l}\text { Sobre semicon. a } \\
\text { 90cm del defecto }\end{array}$ \\
\hline
\end{tabular}

Fuente: Autores, (2017).

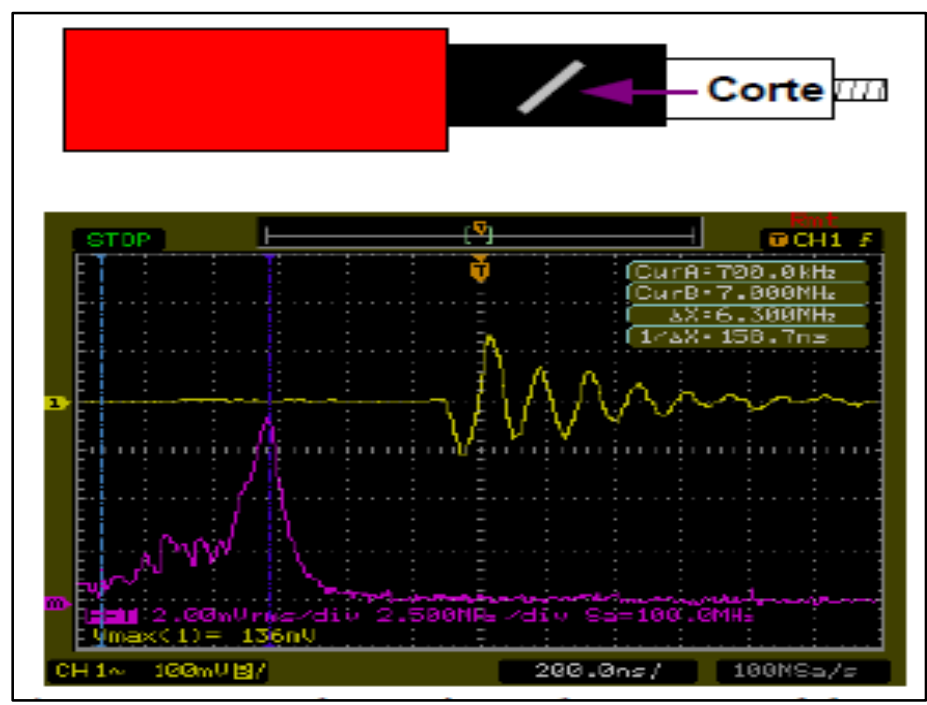

(a) Corte en el semiconductor - cable AT.

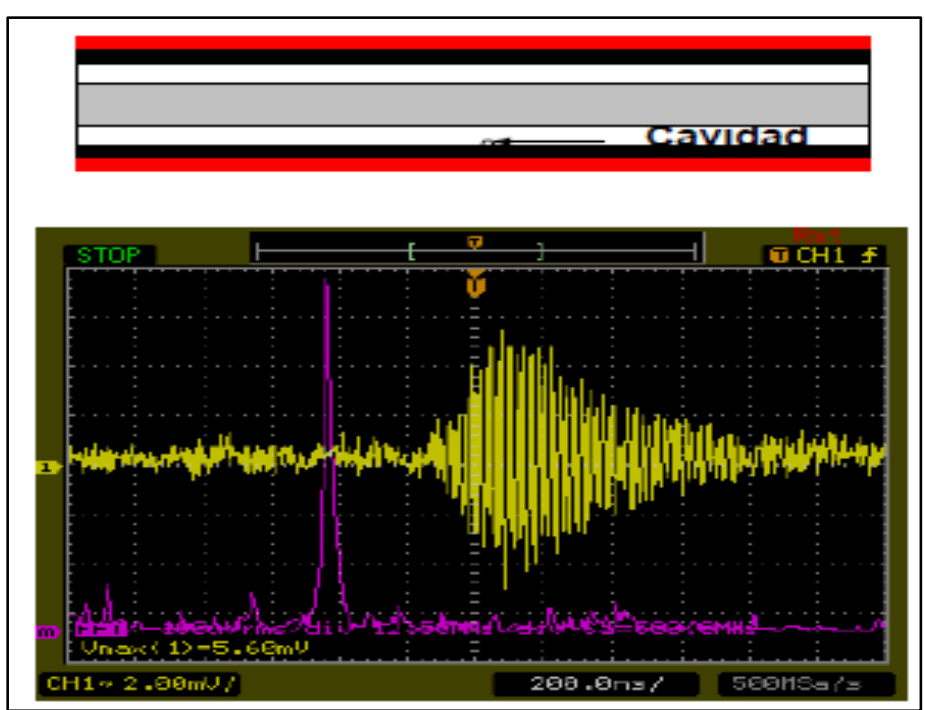

(b) Cavidad $\varnothing=2 \mathrm{~mm}$ en aislamiento - cable MT. 


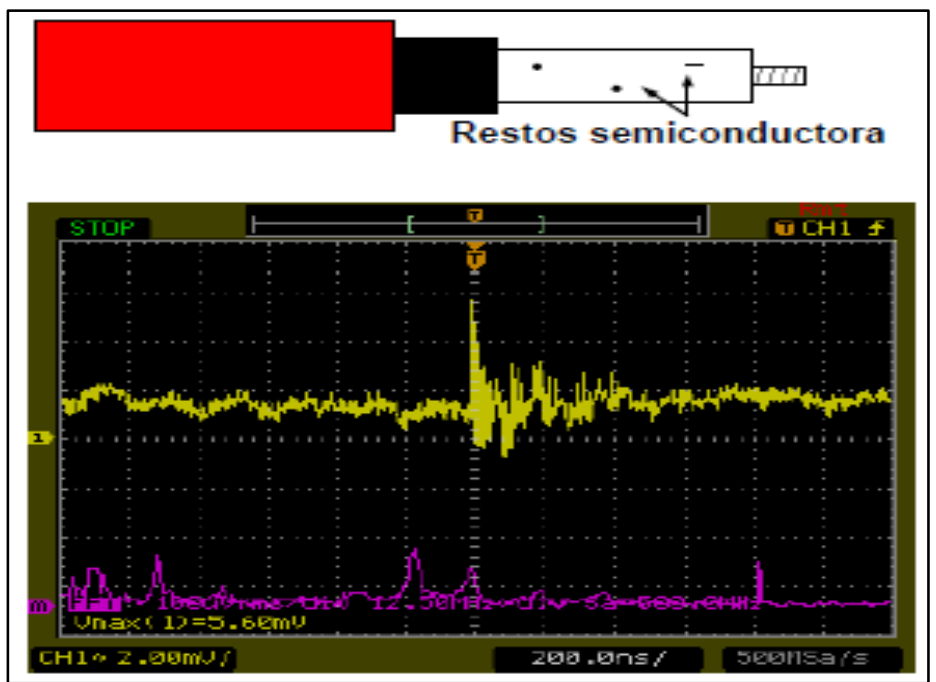

(c) Restos de semiconductor - cable MT.

Figura 9: Modelos de descarga parcial en (a), (b), (c). Fuente: Autores, (2017).

\section{CONCLUSIONES}

- La evolución de los sensores EA se presenta como un refuerzo, o alternativa, a los sistemas eléctricos, en la detección de DP en accesorios de cables, para aplicaciones en línea y en campo.

- El sistema presenta mayor sensibilidad ante descargas internas del aislamiento, tipo cavidad, contenidas debajo el semiconductor.

- El nivel de sensibilidad alcanzado, descargas a partir de $40 \mathrm{pC}$, pueden ser una alternativa en aplicaciones de MT, pero requiere mejorarse para AT.

- La respuesta de los sensores EA, ofrece diferentes formas ante diferentes tipos de defecto. Lo cual da pie a continuar el trabajo con el análisis detallado de las FFT, que también reflejan estas diferencias.

\section{REFERENCIAS}

[1] Working Grup 21.10. Survey on the Service Perfomance on HV AC Cable Systems. Report of Study Committee 21 Insulated Cables, Electra No 137, August 1991, pp. 47-59.

[2] R.M. Bucci, R.V. Rebbapragada, A.J. McElroy, E.A. Chebli, S.Driller; Failure Prediction on Underground Distribution Feeder Cables, IEEE Transactions on Power Delivery, Vol. 9, $\mathrm{N}^{\mathrm{o}}$ 4, October 1994, pp. D2.1-D2.7.

[3] Mashikian, M.S.; Partial discharge location as a diagnostic tool for power cables, Power Engineering Society Winter Meeting, 2000. IEEE, Volume 3, Jan. 2000, pp 1604 - 1608.

[4] Standard IEC 60840: 2004: Power cables with extruded insulation and their accessories for rated voltages above 30 $\mathbf{k V}(\mathbf{U m}=36 \mathrm{kV})$ up to $150 \mathrm{kV}(\mathbf{U m}=170 \mathrm{kV})$ - Test methods and requirements.

[5] Standard IEC 60502-4: 2005: Power cables with extruded insulation and their accessories for rated voltages from $1 \mathbf{~ k V}$ $(\mathbf{U m}=1,2 \mathrm{kV})$ up to $30 \mathrm{kV}(\mathbf{U m}=36 \mathbf{k V})$. Part 4: Test requirements on accessories for cables with rated voltages from 6 $\mathrm{kV}(\mathrm{Um}=7,2 \mathrm{kV})$ up to $30 \mathrm{kV}(\mathrm{Um}=36 \mathrm{kV})$.
[6] Standard IEC 60885-3: 1988: Electrical test methods for electric cables. Part 3: Test methods for partial discharge measurements on lengths of extruded power cables.

[7] Casals Torrens, P. "Sistema en línea para detección preventiva de descargas parciales en accesorios de cables de alta tensión mediante sensores de emisiones acústicas". Director Bosch Tous, R. Tesis doctoral. Universitat Politècnica de Catalunya, Dpto. De Ingeniería Eléctrica, Barcelona, 2008.

[8] Harrold, R.T. Acoustic Theory Applied to the Physics of Electrical Breakdown in Dielectrics. IEEE Transactions on Electrical Insulation, Volume EI-21, nº 5, October 1986, pp. 781 792.

[9] Kreuger, F.H.; Industrial High Voltage. Editorial Delf University Press, Delf-Netherlands, 1991.

[10] Lundgaard, L.E. Partial Discharge - Part XIII: Acoustic Partial Discharge Detection - Fundamental Considerations. IEEE Electrical Insulation Magazine, Vol. 8, $\mathrm{N}^{\mathrm{o}}$ 4, July/August 1992, pp. 25- 31.

[11] Naidu, M.S.; Kamaraju, V.; High Voltage Engineering. Editorial Mc Graw Hill. USA, Segunda edición, 1995.

[12] Mason, J.H.; Enhancing the significance of PD measurements. IEEE Transactions on Dielectrics and Electrical Insulation, Volume 2, Issue 5, Oct. 1995, pp. 876 888. [13] Kinsler, L.E; Frey, A.R.; Coppens, A.B.; Sanders, J.V.; Fundamentals of Acoustic. Editorial John Wiley \& Son, Inc. New York, 4 edition. 2004.

[14] Blackstock, D.T.; Funadamentals of Physical Acoustic. Editorial Wiley-Interscience. New York. 2000.

[15] Boogs, Steven A. Partial Discharge: Overview and SignalGeneration. IEEE Electrical Insulation Magazine, Vol. 6, No 4, July/August 1990, pp. 33-39.

[16] Piche, L.; Pelissou, S.; Crine, J.-P.; Cable insulation density profile determined from ultrasonic velocity measurements. Electrical Insulation Magazine, IEEE, Volume 8, Issue 3, May June 1992, pp 33 - 36.

[17] Suzuki, H.; Kanaoka, M.; Sekii, Y.; Measurements on the frozen strain in XLPE insulated cables using thermo mechanical analysis. Proceedings of the Twenty-First Symposium on Electrical Insulating Materials, 1988.. Sept. 26, 1988 , pp 255 - 258. 